\title{
Peroxidized mineral oil increases the oxidant status of culture media and inhibits in vitro porcine embryo development
}

C. A. Martinez, A. Nohalez, J . J . Ceron, C. P. Rubio, J . Roca, C. Cuello, Heriberto Rodriguez-Martinez, E. A. Martinez and M. A. Gil

The self-archived postprint version of this journal article is available at Linköping University Institutional Repository (DiVA):

http:// urn.kb.se/ resolve?urn=urn:nbn:se:liu:diva-142144

N.B.: When citing this work, cite the original publication.

Martinez, C. A., Nohalez, A., Ceron, J .J ., Rubio, C. P., Roca, J., Cuello, C., Rodriguez-Martinez, H., Martinez, E. A., Gil, M. A., (2017), Peroxidized mineral oil increases the oxidant status of culture media and inhibits in vitro porcine embryo development, Theriogenology, 103, 17-23.

https:// doi.org/ 10.1016/j.theriogenology.2017.07.028

Original publication available at:

https:/ / doi.org/ 10.1016/j.theriogenology.2017.07.028

Copyright: Elsevier

http:// www.elsevier.com/

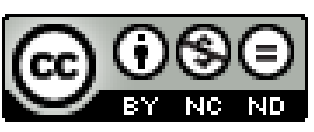


Revised

Peroxidized mineral oil increases the oxidant status of culture media and inhibits in vitro porcine embryo development

CA Martinez ${ }^{\mathrm{a}, \mathrm{b}}$, A Nohalez ${ }^{\mathrm{a}, \mathrm{b}}$, JJ Ceron ${ }^{\mathrm{a}, \mathrm{b}}$, CP Rubio ${ }^{\mathrm{a}}$, J Roca ${ }^{\mathrm{a}, \mathrm{b}}$, C Cuello ${ }^{\mathrm{a}, \mathrm{b}}$, H Rodriguez-

Martinez $^{\mathrm{c}}$, EA Martinez ${ }^{\mathrm{a}, \mathrm{b}^{*}}$, MA Gil $^{\mathrm{a}, \mathrm{b}}$

${ }^{\text {a}}$ Faculty of Veterinary Medicine, International Excellence Campus for Higher Education and Research “Campus Mare Nostrum”, University of Murcia, Murcia, Spain

${ }^{\mathrm{b}}$ Institute for Biomedical Research of Murcia (IMIB-Arrixaca), Murcia, Spain

'Department of Clinical \& Experimental Medicine (IKE), Linköping University, Linköping,

Sweden

*Corresponding author

Emilio A. Martinez

E-mail: emilio@um.es

Tel.: +34 868884734

Fax: +34 868887069 


\begin{abstract}
The use of oils with undetected alterations is a long-recognized problem for in vitro embryo production systems. Since peroxides in oils have been associated with reduced embryo production outcomes, our goals were (1) to evaluate the effects of a batch of mineral oil (MO) that was suspected to be altered on the in vitro production of pig embryos and (2) to determine oil peroxide values throughout culture and the transfer of oxidant agents from oil to culture media. Sunflower oil, which has a completely different chemical composition than MO but a higher oxidative status, and unaltered MO were used as controls.
\end{abstract}

Oocyte maturation, fertilization and embryo development were affected differently depending on the oil overlay used. While the suspected MO was not able to sustain in vitro maturation and fertilization, the oocytes incubated in the presence of sunflower oil were matured and fertilized similarly to those of the unaltered MO group. Moreover, the cleavage rate of presumed zygotes cultured under the suspected MO was severely reduced compared with those cultured under the other oils, and none of the cleaved embryos developed to the blastocyst stage. Although the cleavage rates in the sunflower oil and unaltered MO groups were similar, embryos cultured under sunflower oil also failed to develop to the blastocyst stage. Our results revealed that the suspected MO and sunflower oil had similar levels of peroxides and that these levels were much higher than those of the unaltered MO. The total oxidant status was higher in media incubated under peroxidized oils than in fresh media or media incubated without an oil overlay or under unaltered MO, indicating that oxidant agents were transferred to the incubation media. However, unlike the sunflower oil group, the culture media incubated under the suspected MO had high levels of total oxidant status and low levels of hydrogen peroxide and reactive oxygen species, suggesting the presence of other unknown oxidant agents in that oil. These results indicate that a 
peroxidized MO overlay dramatically decreases embryo production outcomes. This decrease could be associated with the higher peroxide values of the oil but cannot be explained by the levels of hydrogen peroxide and reactive oxygen species transferred from the oil to the culture media. It is likely that different oxidant agent(s) and/or other toxic compounds present in the peroxidized MO are responsible for its damaging effects on oocytes and embryos.

\section{Keywords}

In vitro production of embryos; porcine; mineral oil overlay; peroxidation; in vitro fertilization; in vitro maturation 


\section{Introduction}

Mineral oil is widely used in embryo in vitro production (IVP) systems to cover drops of culture media because among other advantages, it helps to maintain a stable and proper $\mathrm{pH}$ and osmolality in the culture medium [1-5]. This fact is critical since variations in the $\mathrm{pH}$ of the medium might affect embryo metabolism [6,7], whereas osmotic stress may cause DNA and protein alterations that affect cell function [8]. However, the use of an oil overlay may also have adverse effects due to the possibility that toxic contaminants that accumulate in the oil during production, transport or storage may be introduced into the culture medium $[9,10]$ and may negatively affect gametes and embryos. The use of oils with undetected contamination has long been recognized as a problem for embryo cultures. Oil degradation produces alkenals and aldehydes [11], which have been shown to be toxic to embryos when present in culture media [12]. Triton X-100, a nonionic surfactant that is used in research to permeabilize membranes or solubilize proteins, has also been found in oils used for embryo culture where the embryo development was severely inhibited [11]. In another study, zinc was identified as a possible toxic contaminant of silicone oil in mouse embryo cultures [13]. However, peroxides, which produce reactive oxygen species, are possibly the most dangerous contaminants found in altered oils. They can be formed throughout the shelf life of the oil and have been associated with decreased fertilization, cleavage, and blastocyst formation rates in rodents [11,14,15]. Although these findings suggest that peroxidized oil overlays have embryo-toxic effects, the transfer of oxidant compounds from the oil to the culture medium has not been clearly established.

The aims of this study were (1) to evaluate the effects of a batch of mineral oil, which was suspected to be altered (SMO), on the IVP of pig embryos and (2) to determine oil peroxide values (POVs) throughout culture and the transfer of oxidant agents from oil to culture media. 


\section{Materials and methods}

All experiments were performed following the ethical guidelines of the 2010/63/EU EEC

Directive for animal experiments and were approved by the Ethical Committee for

Experimentation with Animals of the University of Murcia, Spain (research code: 183/2015).

\subsection{Collection of cumulus-oocyte complexes (COCs)}

Pre-pubertal gilts of 5.5 to 6 months of age and weighing 90 to $100 \mathrm{~kg}$ were used as COC

donors. The pre-pubertal gilts were sacrificed in a local slaughterhouse, and their ovaries were transported to the laboratory at the University of Murcia at $35{ }^{\circ} \mathrm{C}$ within $1 \mathrm{~h}$ post-collection in 0.9\% NaCl containing $70 \mu \mathrm{g} / \mathrm{mL}$ kanamycin. The COCs were collected with a surgical blade from medium-sized healthy follicles (3 to 6 mm diameter) in Tyrode’s lactate medium supplemented with 10 mM HEPES and 0.1\% polyvinyl alcohol (PVA) [16,17]. Oocytes surrounded by two or more compact cumulus mass complexes and with a dark and granulated cytoplasm were selected for maturation.

\subsection{In vitro maturation (IVM) and assessment of maturation}

The COCs were washed three times in IVM medium. This medium consisted of TCM-199 (Gibco Life Technologies S.A. Barcelona, Spain) supplemented with $0.57 \mathrm{mM}$ cysteine, 0.1\% PVA and $10 \mathrm{ng} / \mathrm{mL}$ epidermal growth factor. Groups of 40 COCs were matured in $500 \mu \mathrm{L}$ of IVM medium supplemented with $10 \mathrm{IU} / \mathrm{mL}$ equine chorionic gonadotrophin (Folligon, Intervet International B.V., Boxxmeer, The Netherlands) and 10 IU/mL human chorionic gonadotrophin (Veterin Corion, Divasa Farmavic, S.A., Barcelona, Spain) for 22 h. The COCs were then 
incubated for an additional $22 \mathrm{~h}$ in the same medium but without hormones. All cultures were incubated under an oil overlay at $38.5{ }^{\circ} \mathrm{C}$ in a humidified atmosphere of $5 \% \mathrm{CO}_{2}$ in air.

To assess the maturation status, the oocytes were fixed in acetic acid:ethanol (1:3) for 72 $\mathrm{h}$ at room temperature and stained with $1 \%$ lacmoid in $45 \%$ acetic acid. The oocytes were considered immature when their chromatin was enclosed in a nuclear membrane (i.e., germinal vesicle stage) or when it was condensed in metaphase I. The oocytes were considered mature when their chromosomes were organized in metaphase and a clear first polar body was visible (MII). Oocytes with a broken cytoplasmic membrane or a cytoplasm with an abnormal appearance were considered to be degenerated. The degenerated, immature and mature oocyte rates were expressed as the ratio of the number of degenerated, immature and MII stage oocytes, respectively, relative to the total number of oocytes evaluated.

\subsection{In vitro fertilization (IVF) and assessment of fertilization status}

At the end of the maturation period, the COCs were denuded by vortexing at 1660 rounds/min for 2 min in IVM medium containing $0.1 \%$ hyaluronidase and washed three times in IVM medium. The oocytes were then washed three times in pre-equilibrated IVF medium [18] supplemented with $2.0 \mathrm{mM}$ caffeine and $0.2 \%$ bovine serum albumin (BSA) and inseminated as described previously [19]. Briefly, the oocytes were placed in groups of 40 in $50-\mu L$ drops of IVF medium covered with oil and kept in the incubator at $38.5^{\circ} \mathrm{C}$ and $5 \% \mathrm{CO}_{2}$ in humidified air for 30 min until sperm were added. Semen cryopreserved in 0.5-mL straws, as described by Carvajal et al. [20], was thawed (two straws for each replicate) at $37^{\circ} \mathrm{C}$ for $20 \mathrm{sec}$ and washed three times at $1900 \times \mathrm{g}$ for 3 min in Dulbecco’s PBS (Gibco, Grand Island, NY) supplemented with $0.1 \%$ BSA. The sperm pellet was then re-suspended in IVF medium, and after being 
appropriately diluted, $50 \mu \mathrm{L}$ of the sperm suspension was added to the drop of IVF medium that contained the oocytes. The spermatozoa:oocyte ratio was 1000:1. The oocytes and spermatozoa were co-incubated at $38.5{ }^{\circ} \mathrm{C}$ in a humidified atmosphere of $5 \% \mathrm{CO}_{2}$ in air for $5 \mathrm{~h}$ under oil.

To evaluate fertilization parameters, presumptive zygotes were fixed and stained as described for the maturation assessment. Presumptive zygotes were classified as penetrated when at least a sperm head and/or male pronucleus, with the corresponding sperm tail, and two polar bodies were visible in their cytoplasm. Oocytes with an abnormal cytoplasm appearance were classified as degenerated. The penetration rate was defined as the ratio of the number of penetrated oocytes relative to the total number of mature oocytes. The monospermic rate was the ratio of the number of oocytes with one female pronucleus, one male pronucleus and two polar bodies relative to the total number of penetrated oocytes. The fertilization efficiency was defined as the ratio of the number of monospermic oocytes relative to the total number of oocytes inseminated. The degeneration rate was the ratio of the number of degenerated presumptive zygotes relative to the total number of oocytes evaluated.

\subsection{In vitro culture (IVC) and assessment of embryonic development parameters}

Following sperm-oocyte co-incubation, groups of 30 presumptive zygotes were washed three times and cultured in $500 \mu \mathrm{L}$ of IVC medium, which consisted of glucose-free pre-equilibrated North Carolina State University culture medium (NCSU-23; [21]) supplemented with $0.4 \%$ BSA, $0.3 \mathrm{mM}$ pyruvate and $4.5 \mathrm{mM}$ lactate, for 2 days. Then, the cleaved embryos were cultured in fresh IVC medium supplemented with $0.4 \%$ BSA and $5.5 \mathrm{mM}$ glucose for an additional 5 days. All embryo cultures were incubated at $38.5{ }^{\circ} \mathrm{C}$ in an atmosphere of $5 \% \mathrm{CO}_{2}$ in air under oil. 
Embryonic development was evaluated morphologically under a stereomicroscope. Embryos with a morphologically abnormal appearance and those that were poorly developed were classified as degenerated embryos. The cleavage rate was defined as the ratio of the number of embryos developed to the 2- to 4-cell stage at day 2 of culture relative to the total number of oocytes inseminated. The blastocyst formation rate was the ratio of the number of 2- to 4-cell embryos that developed to blastocyst stage (an embryo with a clear blastocoel and with good or excellent morphology) at day 7 of culture. The blastocyst efficiency rate was defined as the ratio of the number blastocysts relative to the total number of oocytes inseminated.

\subsection{Analysis of oils and media}

\subsubsection{Determination of the peroxide values in the oils}

The POVs were determined as previously reported [22]. The oil samples were treated with an acetic acid-chloroform solution and a saturated potassium iodide solution and were placed into the burette of a POV meter (DL 25 Titrator, Mettler-Toledo AG, Greifensee, Switzerland). The titration was run against a standard solution of sodium thiosulfate. The POVs were expressed in $\mu \mathrm{mol} / \mathrm{L}$.

2.5.2 Determination of total oxidant status (TOS), hydrogen peroxide $\left(\mathrm{H}_{2} \mathrm{O}_{2}\right)$ and reactive oxygen species (ROS) in the media

The TOS values were assessed as previously described [23] using an Olympus AU600 Automatic Chemistry Analyzer. Briefly, xylenol orange, $\mathrm{NaCl}$ and glycerol in a $\mathrm{H}_{2} \mathrm{SO}_{4}$ solution were incubated with the sample for $3 \mathrm{~min}$. Subsequently, ferrous ion and o-dianisidine in $\mathrm{H}_{2} \mathrm{SO}_{4}$ 
were added to the reaction. The change in absorbance was monitored, and the results were calculated using a standard curve of $\mathrm{H}_{2} \mathrm{O}_{2}$ solutions and expressed in $\mu \mathrm{mol} / \mathrm{L}$.

$\mathrm{H}_{2} \mathrm{O}_{2}$ was detected as described by Rhee et al. [24]. Briefly, the samples were incubated for 15 min at $22^{\circ} \mathrm{C}$ with horseradish peroxidase (HRP) and 3,5,3'5'-tetramethylbenzidine (TBM) in sodium phosphate buffer, and the reaction was then terminated by the addition of sulfuric acid. The $\mathrm{H}_{2} \mathrm{O}_{2}$ concentration of the samples was estimated based on the molar extinction coefficient of diamine using a microplate reader (Powerwave XS, Biotek Instruments, Carson City, NV). The results were expressed in $\mu \mathrm{mol} / \mathrm{L}$.

ROS were measured by a luminol-mediated chemiluminescence assay [25]. Luminol containing HRP was added to the medium and saline solution. The ROS values were assessed using a microplate reader (Victor 21420 Multilabel Counter; PerkinElmer, Finland) and expressed in photon count per second (cps). A negative or background control was prepared by adding saline solution instead of sample.

\subsection{Experimental design}

In the present study, we compared the effects of an SMO overlay (Cat. no. M8410, lot no. MKBW2313V; Sigma-Aldrich Co., St. Louis, MO, USA) on IVM, IVF and embryonic development. This oil had performed poorly in previous experiments. Sunflower oil (SFO), which has a completely different chemical composition than MO but a high oxidative status, and unaltered MO (UMO; Cat. no. M8410, lot no. MKBX6122V; Sigma-Aldrich Co., St. Louis, MO, USA) were used as controls.

To evaluate the maturation and fertilization parameters, immature oocytes were matured and fertilized in media covered with SMO, SFO or UMO in four replicates. At $44 \mathrm{~h}$ of 
maturation, a random subset of the oocytes $(n=460)$ was fixed and stained to determine the percentage of oocytes reaching the MII stage. Eighteen hours post-insemination, the remaining oocytes ( $n=455)$ were fixed and stained to determine the degeneration, penetration and monospermic rates, as well as the fertilization efficiency. In another set of experiments, immature oocytes $(n=316)$ were matured and fertilized under UMO and then cultured under SMO for seven days to evaluate the effects of SMO on the embryonic development. Immature oocytes matured, fertilized and cultured under SFO $(n=305)$ or UMO $(n=413)$ were used as controls.

To evaluate the oxidation status in oils and media, 500- $\mu \mathrm{L}$ drops of IVM or IVC medium covered with SMO, SFO or UMO were incubated for 22 h (IVM medium) or for 2 and 5 days (IVC medium) in the absence of oocytes or embryos. At the end of each incubation period, the oil cover was transferred to 5 mL-tubes for analysis. The medium was carefully transferred to 1 mL-tubes, avoiding cross-contamination with the oil. This process was repeated three times. The assessments were performed in three replicates immediately after sample collection.

\subsection{Statistical analysis}

Binary variable data (degeneration, maturation, penetration, monospermy, cleavage, blastocyst formation and efficiencies) were obtained by calculating the percentage in every drop of each group and in each of the four replicates and are expressed as the mean \pm standard deviation (SD). Continuous variables are expressed as the mean \pm SD of three replicates. Statistical analysis was performed using the IBM SPSS 19 Statistics package (SPSS, Chicago, IL, USA). Variables were analyzed using the Kolmogorov-Smirnov test for normally distributed data. Differences between means of more than two groups were determined by mixed-model analysis of variance 
(ANOVA) followed by the Bonferroni post hoc test. Pairwise comparisons of the means were performed using Student's t-tests. Differences were considered significant at $\mathrm{P}<0.05$.

\section{Results}

The maturation rates of oocytes cultured under each of these oils are presented in Fig. 1. The SMO greatly increased $(\mathrm{P}<0.02)$ the proportion of oocytes with clear signs of degeneration at the end of the maturation period (87.2 $\pm 4.6 \%)$ compared with the SFO (15.2 $\pm 2.4 \%)$ and UMO (8.5 $\pm 2.4 \%)$ control groups. Consequently, there was a significant reduction $(\mathrm{P}<0.001)$ in MII formation rates in oocytes matured in the presence of SFO $(4.2 \pm 2.2 \%)$ compared with oocytes matured under SFO or UMO (74.5 $\pm 7.1 \%$ and $82.7 \pm 7.6 \%$, respectively). The SMO overlay had no significant influence on the percentage of oocytes arrested at the germinal vesicle stage at 44 h of maturation.

All surviving oocytes from the SMO group were degenerated at $18 \mathrm{~h}$ post-insemination, so it was not possible to evaluate IVF parameters. By contrast, although oocytes matured and fertilized under SFO had increased $(\mathrm{P}<0.03)$ rates of degeneration at $18 \mathrm{~h}$ post-insemination compared with the UMO group (13.2 $\pm 5.4 \%$ vs. $5.0 \pm 2.2 \%$ ), no differences were observed in rates of sperm penetration, monospermy and IVF efficiency between these two groups (Fig. 2).

To evaluate the effects of SFO on embryonic development, immature oocytes were matured and fertilized under UMO and then cultured for 7 days under SMO. Embryonic development data are presented in Fig. 3. There was no difference in cleavage rate between the SFO and UMO groups (62.9 $\pm 7.8 \%$ and $59.6 \pm 6.9 \%$, respectively), but the proportion of cleaved embryos derived from presumed zygotes cultured under SMO (25.0 $4.9 \%)$ was dramatically decreased $(\mathrm{P}<0.001)$. The blastocyst formation rates achieved in the UMO group (33.1 $\pm 7.3 \%)$ 
were within the normal range in our laboratory. However, no blastocyst formation was observed upon culture of presumed zygotes under SMO or SFO.

The POVs in oils incubated with IVM or IVC medium are presented in Tables 1 and 2. At $0 \mathrm{~h}$ of incubation, the POVs of SMO and SFO were approximately 100 -fold greater than those of UMO. These differences in POVs were maintained throughout the incubation period. Although no differences in POVs were observed between SMO and SFO at $0 \mathrm{~h}$ of incubation, the POVs were close to 1.5-fold higher $(\mathrm{P}<0.03)$ in $\mathrm{SMO}$ than in SFO after incubation, regardless of culture time and medium.

The oxidant levels of IVM and IVC media incubated under the different oils are presented in Tables 3 and 4. When we evaluated the oxidative status of fresh IVM and IVC media ( $0 \mathrm{~h}$ of incubation), the levels of TOS and $\mathrm{H}_{2} \mathrm{O}_{2}$ were below the limit of detection. These values remained below the limit of detection at $22 \mathrm{~h}$ of incubation (IVM medium), while the TOS levels increased above this limit at day 2 or 5 of incubation (IVC medium). The ROS values in fresh IVM medium $(4872.5 \pm 57.6 \mathrm{cps})$ increased $(\mathrm{P}<0.05)$ to $5046.7 \pm 167.6 \mathrm{cps}$ at $22 \mathrm{~h}$ of incubation in the absence of an oil overlay. A similar pattern was observed for IVC medium, in which the ROS values increased $(\mathrm{P}<0.001)$ from $3447.0 \pm 40.8 \mathrm{cps}$ at $0 \mathrm{~h}$ of incubation to $5483.3 \pm 90.1$ cps at 2 days of incubation in the absence of an oil overlay. The TOS, $\mathrm{H}_{2} \mathrm{O}_{2}$ and ROS values of IVM and IVC media incubated under UMO were similar to those observed in the media incubated without an oil overlay, regardless of the incubation time. By contrast, IVM medium or IVC medium incubated under SMO had higher values $(\mathrm{P}<0.02)$ of TOS than media incubated under $\mathrm{UMO}$, while the $\mathrm{H}_{2} \mathrm{O}_{2}$ and ROS values were not significantly different. Media incubated under SFO had higher $(\mathrm{P}<0.02)$ levels of TOS, $\mathrm{H}_{2} \mathrm{O}_{2}$ and $\mathrm{ROS}$ than media incubated without an oil overlay or medium covered with UMO at any incubation time. 


\section{Discussion}

The results from this study clearly demonstrate that porcine oocytes and embryos did not survive IVC due to one batch of mineral oil that had been used as an oil overlay for in vitro maturation, fertilization and embryo culture. This study also supports previous findings in mice regarding the negative effects of peroxide-contaminated oil on in vitro fertilization and embryo development success [14]. In addition, we also demonstrate the transfer of oxidant molecules from the oil overlay into the incubation media.

This study was conceived because in our routine experiments, we noticed a sudden decrease in maturation, fertilization and embryonic development rates compared with our previous outcomes. This problem became more pronounced over time. We suspected that the problem could be related to the use of a new batch of mineral oil, since no modifications in laboratory environmental conditions or methodological procedures had been introduced. Because peroxides are one of the most damaging contaminants found in oil $[11,14,15]$, we decided to investigate the POV of the SMO and the possible transfer of oxidant agents from the oil into the incubation media. Because gametes and embryos produce many oxidant agents via their metabolic processes (reviewed by Takahashi [26]), in our study, the incubation of medium under oil was performed in the absence of oocytes or embryos. Therefore, the oxidative status of each medium was determined in the absence of metabolic processes. Additionally, we used SFO and UMO for comparison. We used SFO for two main reasons: First, SFO, which contains mainly saturated, monounsaturated and polyunsaturated fatty acids [27,28], has a completely different chemical composition than mineral oil; and second, the POVs of SFO are usually much higher $[27,29]$ than those of UMO. 
Our results revealed that SMO and SFO had similar levels of peroxides at $0 \mathrm{~h}$ of incubation and that in both cases, these levels were much higher than those found in UMO. Additionally, the TOS values were higher in the media incubated under peroxidized oils compared with those obtained in fresh media (0 h of incubation) or media incubated without oil overlay or under UMO, which indicates a clear transfer of oxidant molecules from the oils into the incubation media.

Despite these findings, SMO and SFO affected oocyte maturation, fertilization and development differently. Thus, while SMO was not able to sustain in vitro maturation and fertilization, with all oocytes being degenerated at $18 \mathrm{~h}$ post-insemination, only $15 \%$ of oocytes degenerated in the presence of SFO, and the remaining oocytes were fertilized similarly to those of the UMO group. Moreover, the cleavage rates of presumed zygotes cultured under SMO were severely reduced compared with the SFO group, and none of the cleaved embryos developed to the blastocyst stage in the SMO group. The harmful effects of SFO on embryonic development were not observed at $48 \mathrm{~h}$ of culture, since the cleavage rates in that group were no different from those observed in the UMO group. However, like in the SMO group, all embryos cultured under SFO failed to develop to the blastocyst stage.

The reasons for these differences between SMO and SFO are not clear. It is possible that the increase observed in the POV of SMO both at $22 \mathrm{~h}$ of maturation and at day 2 of culture compared with that of SFO could be responsible for the differences in degeneration and cleavage rates, respectively, between the oils. Supporting this speculation, it has been demonstrated that embryonic degeneration is accelerated with increased levels of oil peroxidation [14]. However, this is not necessarily the case, since the IVM or IVC medium incubated under SMO had similar levels of $\mathrm{H}_{2} \mathrm{O}_{2}$ and ROS as the UMO group and lower levels than the SFO group. Moreover, 
despite the huge differences in POV, $\mathrm{H}_{2} \mathrm{O}_{2}$ and ROS levels between the SFO and UMO groups, no differences in maturation rate, fertilization parameters or cleavage rate were observed between these groups. These findings indicate that $\mathrm{H}_{2} \mathrm{O}_{2}$ and $\mathrm{ROS}$ were not directly responsible for the destructive effects of SMO on oocytes and embryos.

Interestingly, the media incubated under SMO had higher levels of TOS than the media incubated under UMO. However, the results obtained regarding TOS levels in IVM and IVC media incubated under SMO seem contradictory, because these values were lower at $22 \mathrm{~h}$ of maturation and higher at 2 days and 5 days of embryo culture than those obtained in the SFO group. This apparent discrepancy can be explained by two reasons: First, although the main TOS contributors are hydrogen peroxide and lipid hydroperoxides [23], other components that contribute to TOS values remain unknown, which could explain the high TOS levels in the presence of low levels of $\mathrm{H}_{2} \mathrm{O}_{2}$ and ROS in the SMO group; and second, some of these unknown compounds could also interact differently depending on the medium composition, resulting in the differences in TOS values observed in the media in this study. This hypothesis is supported by previous findings reporting that the generation of oxidant agents depends on the composition of the culture medium [30-32] and that specific substances, including ionic elements and serum albumin, present in the culture medium can increase the oxidative status of the medium [33,34]. Overall, our results suggest that the negative effects of SFO could be due, at least in part, to the increased $\mathrm{H}_{2} \mathrm{O}_{2}$ and ROS values in the culture media, which are probably associated with the higher POVs conserved in SFO. By contrast, unknown oxidant compounds detected by TOS analysis could be responsible for the poor performance of SMO. Nevertheless, the possibility that other toxic compounds released from SMO could be implicated in its damaging effects 
should be considered. In accordance with this hypothesis, aldehydes and alkenals, which are embryo toxic [12], have also been identified in peroxidized oils [11].

\subsection{Conclusions}

In conclusion, this manuscript reports a case in which alterations in the state of mineral oil used to overlay culture media dramatically decreased IVP outcomes. This decrease could be related to the higher POV concentration of the suspected oil but cannot be explained by the levels of $\mathrm{H}_{2} \mathrm{O}_{2}$ and ROS in the culture media. It is likely that different oxidant agent(s) and/or other toxic compounds present in the peroxidized mineral oil are responsible for its damaging effects on oocytes and embryos. Appropriate conservation and control procedures, including measuring the POV in the oils used for IVP culture media, are recommended.

\section{Acknowledgements}

The authors are grateful to AIM Iberica (Murcia, Spain) and El Pozo (Murcia, Spain) for supplying the boar ejaculates and the ovaries, respectively, used in this study.

\section{Funding}

This study was supported by the Ministry of Economy and Competitiveness (Madrid, Spain) and the European Regional Development Fund (grant numberAGL2015-69735-R); and th Seneca Foundation, Murcia, Spain (grant number 19892/GERM/15). The Ministry of Economy and Competitiveness is also acknowledged for its grant-based support to C.A. Martinez and A. Nohalez (BES-2013-064087 and BES-2013-064069, respectively). 


\section{Role of the funding source}

Funding sources did not have any involvement in the study design, in the collection, analysis and interpretation of data, in the writing of the report, and in the decision to submit the article for publication.

\section{Author contributions}

C.A.M., H.R.-M., E.A.M. and M.A.G. conceived the study. C.A.M., A.N., I.P., J.R., C.C., E.A.M. and M.A.G. performed the experiments related to embryo production. J.J.C and C.P.R. collected and interpreted the data regarding oil and medium analyses. C.A.M., H.R.-M., E.A.M. and M.A.G. wrote the manuscript. All authors reviewed the manuscript.

\section{Declaration of interest}

None of the authors have any conflicts of interest to declare. 


\section{References}

[1] Brinster RL. In vitro culture of mammalian embryos. J Anim Sci 1968;27:1-14.

[2] Boatman DE. In vitro growth of non-human primate pre- and peri-implantation embryos. In: Bavister BD, editor. The mammalian preimplantation embryo: regulation of growth and differentiation in vitro, New York: Plenum Press; 2009. p. 273-303.

[3] Miller KF, Goldberg JM, Collins RL. Covering embryo cultures with mineral oil alters embryo growth by acting as a sink for an embryotoxic substance. J Assist Reprod Genet $1994 ; 11: 342-5$.

[4] Gardner DK, Lane M. Ex vivo early embryo development and effects on gene expression and imprinting. Reprod Fertil Dev 2005;17:361-70.

[5] Tae JC, Kim EY, Lee WD, Park SP, Lim JH. Sterile filtered paraffin oil supports in vitro developmental competence in bovine embryos comparable to co-culture. J Assist Reprod Genet 2006;23:121-7.

[6] Edwards LJ, Williams DA, Gardner DK. Intracellular $\mathrm{pH}$ of the preimplantation mouse embryo: effects of extracellular pH and weak acids. Mol Reprod Dev 1998;50:434-42.

[7] Lane M, Gardner DK. Regulation of ionic homeostasis by mammalian embryos. Semin Reprod Med 2000;18:195-204.

[8] Burg MB, Ferraris JD, Dmitrieva NI. Cellular response to hyperosmotic stresses. Physiol Rev 2007;87:1441-74.

[9] Lee S, Cho M, Kim E, Kim T, Lee C, Han J, et al. Renovation of a drop embryo cultures system by using refined mineral oil and the effect of glucose and/or hemoglobin added to a serum-free medium. J Vet Med Sci 2004;66:63-6. 
[10] Morbeck DE, Leonard PH. Culture systems: mineral oil overlay. Methods Mol Biol 2012;912:325-31.

[11] Morbeck DE, Khan Z, Barnidge DR, Walker DL. Washing mineral oil reduces contaminants and embryotoxicity. Fertil Steril 2010;94:2747-52.

[12] Hall J, Gilligan A, Schimmel T, Cecchi M, Cohen J. The origin, effects and control of air pollution in laboratories used for human embryo culture. Hum Reprod 1998;13:146-55.

[13] Erbach GT, Bhatnagar P, Baltz JM, Biggers JD. Zinc is a possible toxic contaminant of silicone oil in microdrop cultures of preimplantation mouse embryos. Hum Reprod 1995;10:3248-54.

[14] Otsuki J, Nagai Y, Chiba K. Peroxidation of mineral oil used in droplet culture is detrimental to fertilization and embryo development. Fertil Steril 2007;88:741-3.

[15] Otsuki J, Nagai Y, Chiba K. Damage of embryo development caused by peroxidized mineral oil and its association with albumin in culture. Fertil Steril 2009;91:1745-9.

[16] Funahashi H, Ekwall H, Rodriguez-Martinez H. Zona reaction in porcine oocytes fertilized in vivo and in vitro as seen with scanning electron microscopy. Biol Reprod 2000;63:1437-42.

[17] Martinez EA, Angel MA, Cuello C, Sanchez-Osorio J, Gomis J, Parrilla I, et al. Successful non-surgical deep uterine transfer of porcine morulae after 24 hour culture in a chemically defined medium. PLoS One 2014;9:e104696.

[18] Abeydeera LR, Day BN. In vitro penetration of pig oocytes in a modified Tris-buffered medium: effect of BSA, caffeine and calcium. Theriogenology 1997;48:537-44. 
[19] Gil MA, Abeydeera LR, Day BN, Vazquez JM, Roca J, Martinez EA. Effect of the volume of medium and number of oocytes during in vitro fertilization on embryo development in pigs. Theriogenology 2003;60:767-76.

[20] Carvajal G, Cuello C, Ruiz M, Vázquez JM, Martinez EA, Roca J. Effects of centrifugation before freezing on boar sperm cryosurvival. J Androl 2004;25:389-96.

[21] Petters RM, Wells KD. Culture of pig embryos. J Reprod Fertil Suppl 1993;48:61-73.

[22] Association of Analytical Communities. Official methods of analysis of Association of Official Analytical Chemists. Arlington, VA, USA: Association of Analytical Communities; 1995.

[23] Erel O. A new automated colorimetric method for measuring total oxidant status. Clin Biochem 2005;38:1103-11.

[24] Rhee SG, Chang TS, Jeong W, Kang D. Methods for detection and measurement of hydrogen peroxide inside and outside of cells. Mol Cells 2010;29:539-49.

[25] Vong L, Lorentz RJ, Assa A, Glogauer M, Sherman PM. Probiotic Lactobacillus rhamnosus inhibits the formation of neutrophil extracellular traps. J Immunol 2014;192:1870-7.

[26] Takahashi M. Oxidative stress and redox regulation on in vitro development of mammalian embryos. J Reprod Dev 2012;58:1-9.

[27] Pal US, Patra RK, Sahoo NR, Bakhara CK, Panda MK. Effect of refining on quality and composition of sunflower oil. J Food Sci Technol 2015;52:4613-8.

[28] Orsavova J, Misurcova L, Ambrozova JV, Vicha R, Mlcek J. Fatty acids composition of vegetable oils and its contribution to dietary energy intake and dependence of cardiovascular mortality on dietary intake of fatty acids. Int J Mol Sci 2015;16:12871-90. 
[29] Crapiste GH, Brevedan MIV, Carelli AA. Oxidation of sunflower oil during storage. J Am Oil Chem Soc 1999;76:1437.

[30] Agarwal A, Said TM, Bedaiwy MA, Banerjee J, Alvarez JG. Oxidative stress in an assisted reproductive techniques setting. Fertil Steril 2006;86:503-12.

[31] Martin-Romero FJ, Miguel-Lasobras EM, Dominguez-Arroyo JA, Gonzalez-Carrera E, Alvarez IS. Contribution of culture media to oxidative stress and its effect on human oocytes. Reprod Biomed Online 2008;17:652-61.

[32] Shih Y-F, Lee T-H, Liu C-H, Tsao H-M, Huang C-C, Lee M-S. Effects of reactive oxygen species levels in prepared culture media on embryo development: a comparison of two media. Taiwan J Obstet Gynecol 2014;53:504-8.

[33] Shannon P. Factors affecting semen preservation and conception rates in cattle. J Reprod Fertil 1978;54:519-27.

[34] Nasr-Esfahani M, Johnson MH, Aitken RJ. The effect of iron and iron chelators on the invitro block to development of the mouse preimplantation embryo: BAT6 a new medium for improved culture of mouse embryos in vitro. Hum Reprod 1990;5:997-1003. 


\section{Figure legends}

Fig. 1. In vitro maturation efficiency of oocytes incubated in maturation medium covered with suspected mineral oil (SMO; $n=150)$, sunflower oil (SFO; $n=157$ ) or unaltered mineral oil (UMO; $\mathrm{n}=153$ ). The different letters within each variable indicate significant differences (a,b,c: $\mathrm{P}<0.03$; d,e: $\mathrm{P}<0.001)$. The data are presented as the mean $\pm \mathrm{SD}$ of four replicates.

Fig. 2. In vitro fertilization parameters of oocytes matured and fertilized in media covered with suspected mineral oil (SMO; $n=156)$, sunflower oil (SFO; $n=152$ ) or unaltered mineral oil (UMO; n=147). Penetration: percentage of oocytes penetrated/total inseminated oocytes. Monospermy: percentage of oocytes containing one female pronucleus, one male pronucleus and two polar bodies/total penetrated oocytes. Fertilization efficiency: percentage of monospermic oocytes/total inseminated oocytes. The different letters within each variable indicate significant differences $(\mathrm{P}<0.001)$. The data are presented as the mean \pm SD of four replicates.

Fig. 3. Embryonic development of presumed zygotes cultured for seven days in culture medium covered with suspected mineral oil (SMO; n=316), sunflower oil (SFO; $n=305)$ or unaltered mineral oil (UMO; n=413). Presumed zygotes from the SMO group were matured and fertilized in media covered with UMO. Blastocyst formation: percentage of blastocysts/total cleaved embryos. Blastocyst efficiency: percentage of blastocysts/total cultivated oocytes. The different letters within each variable indicate significant differences $(\mathrm{P}<0.001)$. The data are presented as the mean $\pm \mathrm{SD}$ of four replicates. 
Tables

Table 1. Peroxide values $(\mu \mathrm{mol} / \mathrm{L})$ in oils incubated with maturation medium for $22 \mathrm{~h}$.

\begin{tabular}{lcc}
\hline & \multicolumn{2}{c}{ Time of culture (h) } \\
\cline { 2 - 3 } Group & 0 & 22 \\
\hline Suspected MO & $638.4 \pm 75.6^{\mathrm{aA}}$ & $803.7 \pm 55.6^{\mathrm{aB}}$ \\
Sunflower oil & $565.7 \pm 87.7^{\mathrm{a}}$ & $626.1 \pm 35.2^{\mathrm{b}}$ \\
Unaltered MO & $6.4 \pm 0.6^{\mathrm{b}}$ & $6.1 \pm 0.1^{\mathrm{c}}$ \\
\hline
\end{tabular}

MO: mineral oil.

a,b,c Different letters in the same column indicate differences $(\mathrm{P}<0.001)$.

A,B, Different letters in the same row indicate differences $(\mathrm{P}<0.05)$.

Data are presented as the mean \pm SD (three replicates). 
Table 2. Peroxide values ( $\mu \mathrm{mol} / \mathrm{L}$ ) in oils incubated with embryo culture medium for 2 and 5 days.

\section{Time of culture (days)}

\begin{tabular}{lccc}
\cline { 2 - 4 } Group & 0 & 2 & 5 \\
\hline Suspected MO & $638.4 \pm 75.6^{\mathrm{a}}$ & $820.3 \pm 106.0^{\mathrm{a}}$ & $789.6 \pm 73.2^{\mathrm{a}}$ \\
Sunflower oil & $565.7 \pm 87.7^{\mathrm{a}}$ & $592.7 \pm 82.3^{\mathrm{b}}$ & $585.0 \pm 28.1^{\mathrm{b}}$ \\
Unaltered MO & $6.4 \pm 0.6^{\mathrm{b}}$ & $6.8 \pm 0.7^{\mathrm{c}}$ & $6.6 \pm 0.4^{\mathrm{c}}$ \\
\hline
\end{tabular}

MO: mineral oil.

a,b,c Different letters in the same column indicate differences $(\mathrm{P}<0.008)$.

Data are presented as the mean \pm SD (three replicates). 
Table 3. Total oxidant status (TOS; $\mu \mathrm{mol} / \mathrm{L})$, hydrogen peroxide $\left(\mathrm{H}_{2} \mathrm{O}_{2} ; \mu \mathrm{mol} / \mathrm{L}\right)$ and reactive oxygen species (ROS; cps) levels in maturation media covered with the different oils and incubated for $22 \mathrm{~h}$.

\begin{tabular}{l}
\multicolumn{5}{c}{ Time of culture (hours) } \\
\cline { 2 - 5 } Oil cover \\
\cline { 2 - 5 }
\end{tabular}


1 Table 4. Total oxidant status (TOS; $\mu \mathrm{mol} / \mathrm{L})$, hydrogen peroxide $\left(\mathrm{H}_{2} \mathrm{O}_{2} ; \mu \mathrm{mol} / \mathrm{L}\right)$ and reactive oxygen species (ROS; cps) levels in

2 embryo culture media covered with the different oils and incubated for 2 and 5 days.

\begin{tabular}{|c|c|c|c|c|c|c|c|c|c|}
\hline \multirow[b]{3}{*}{ Oil cover } & \multicolumn{9}{|c|}{ Time of culture (days) } \\
\hline & \multicolumn{3}{|c|}{0} & \multicolumn{3}{|c|}{2} & \multicolumn{3}{|c|}{5} \\
\hline & TOS & $\mathrm{H}_{2} \mathrm{O}_{2}$ & ROS & TOS & $\mathrm{H}_{2} \mathrm{O}_{2}$ & ROS & TOS & $\mathrm{H}_{2} \mathrm{O}_{2}$ & ROS \\
\hline Suspected & - & - & - & $16.6 \pm 0.2^{\mathrm{a}}$ & $<\mathrm{LOD}$ & $5622.7 \pm 231.0^{\mathrm{a}}$ & $15.7 \pm 0.4^{\mathrm{a}}$ & $<\mathrm{LOD}$ & $5846.0 \pm 574.1^{\mathrm{a}}$ \\
\hline \multicolumn{10}{|l|}{ MO } \\
\hline Sunflower oil & - & - & - & $4.6 \pm 0.4^{\mathrm{b}}$ & $4.2 \pm 0.3$ & $16481.7 \pm 743.2^{b}$ & $4.3 \pm 0.2^{b}$ & $4.3 \pm 0.4$ & $15639.3 \pm 705.0^{\mathrm{b}}$ \\
\hline Unaltered MO & - & - & - & $2.8 \pm 0.1^{\mathrm{c}}$ & $<\mathrm{LOD}$ & $5831.0 \pm 156.4^{\mathrm{a}}$ & $2.9 \pm 0.1^{\mathrm{c}}$ & $<\mathrm{LOD}$ & $6057.7 \pm 194.4^{\mathrm{a}}$ \\
\hline None & $<\mathrm{LOD}$ & $<\mathrm{LOD}$ & $3447.0 \pm 40.8^{\mathrm{A}}$ & $2.3 \pm 0.1^{\mathrm{c}}$ & $<\mathrm{LOD}$ & $5483.3 \pm 90.1^{\mathrm{aB}}$ & $2.3 \pm 0.3^{\mathrm{c}}$ & $<\mathrm{LOD}$ & $5544.7 \pm 80.2^{\mathrm{aB}}$ \\
\hline
\end{tabular}

3 MO: mineral oil; LOD: Limit of detection (1.41 $\mu \mathrm{mol} / \mathrm{L}$ for TOS and $1.79 \mu \mathrm{mol} / \mathrm{L}$ for $\left.\mathrm{H}_{2} \mathrm{O}_{2}\right)$.

$4 \quad{ }^{\mathrm{a}, \mathrm{b}}$ Different letters in the same column indicate differences $(\mathrm{P}<0.02)$.

5 A,B, Different letters in the same row indicate differences $(\mathrm{P}<0.001)$.

6 Data are presented as the mean \pm SD (three replicates). 


\section{$\because$ SMO SFO UMO}

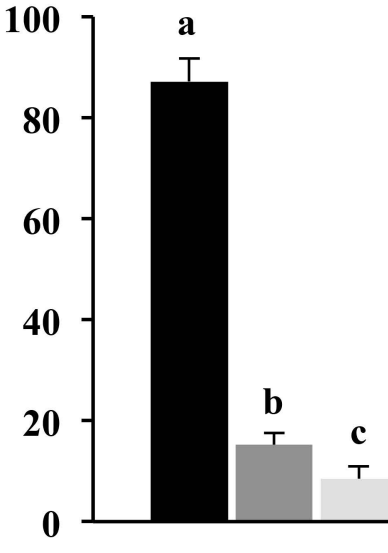

Degenerate
Immature

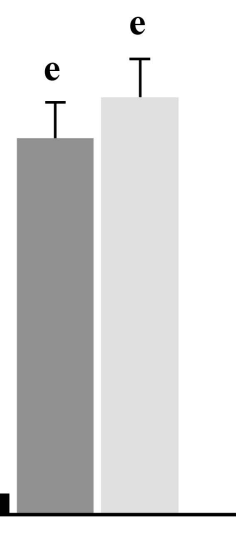

Mature

Oocytes 

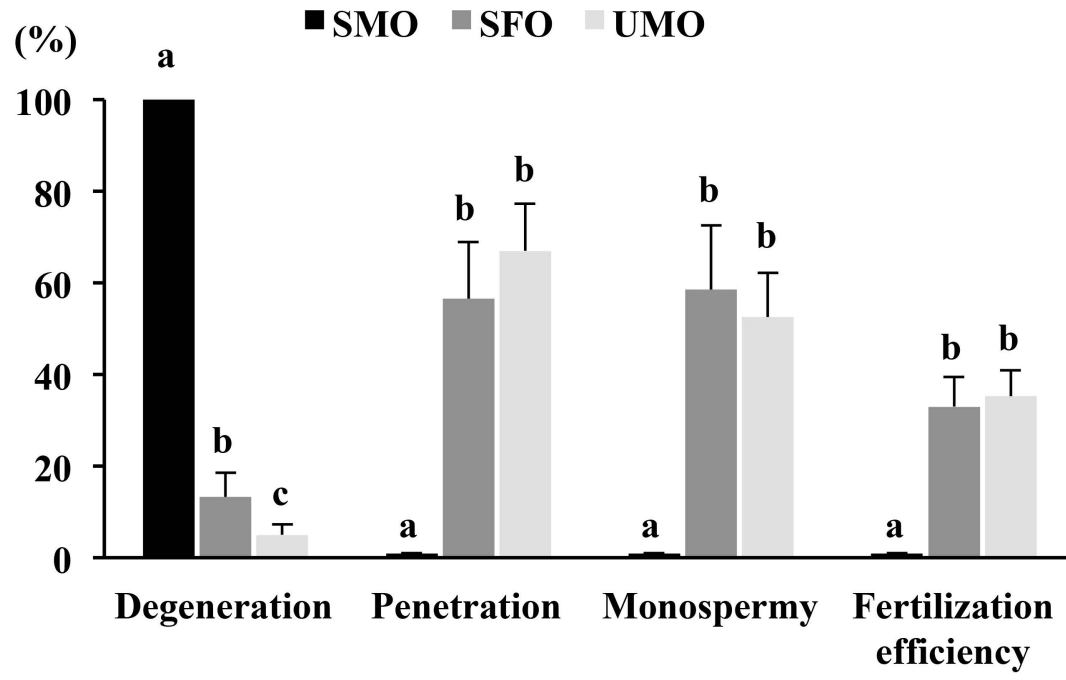


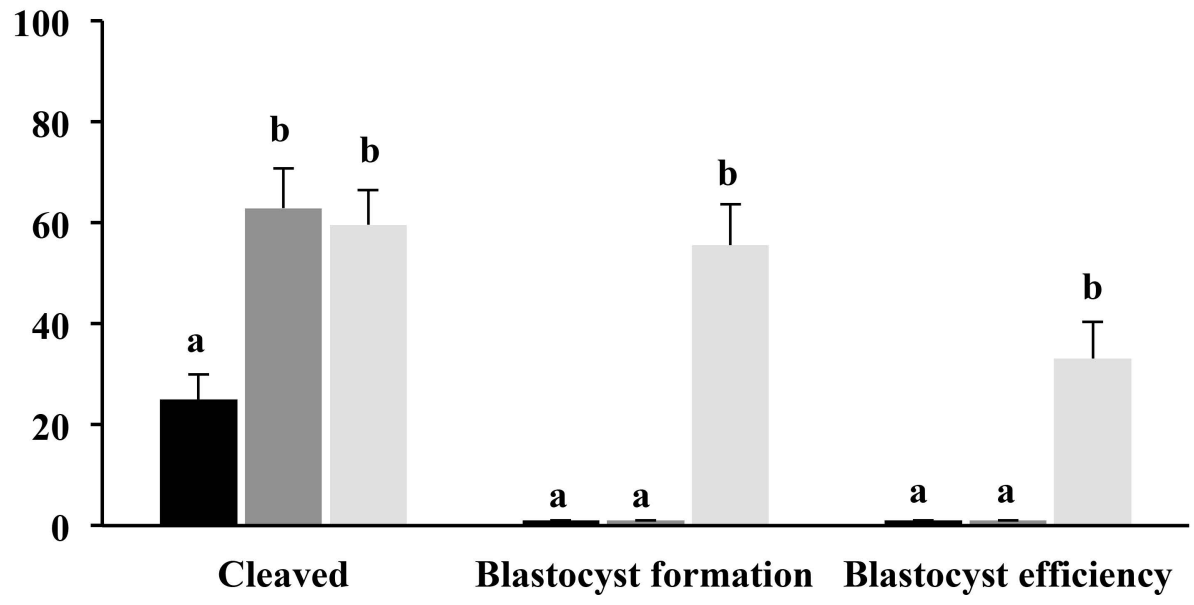

Embryonic development 